\title{
Perfiles Identitarios de Trabajadores de Grandes Empresas del Retail en Santiago de Chile: Aportes Psicosociales a la Comprensión de las Identidades Laborales
}

\author{
Identity Profiles of Workers in Large Retail Companies in \\ Santiago, Chile: Psychosocial Contributions \\ to the Understanding of Labor Identities
}

\author{
Antonio Stecher \\ Universidad Diego Portales
}

\begin{abstract}
Se presentan los resultados de un estudio cualitativo sobre las identidades laborales de trabajadores de tiendas de grandes y modernizadas empresas del retail (supermercados, tiendas por departamento, tiendas para el mejoramiento del hogar) en Santiago de Chile. Se analizan los relatos de una muestra no probabilística intencionada opinática de 18 trabajadores divididos en 4 grupos según género y generación: 5 mujeres y 4 hombres menores de 40 años y 5 mujeres y 4 hombres mayores de 45 años. La información, generada a través de entrevistas en profundidad, fue analizada con el modelo de análisis narrativo holístico y de contenido, buscando reconstruir el sentido, la trama y la orientación global de las narrativas identitarias de los trabajadores. Se presentan 3 perfiles identitarios o tipos de identidad laboral, los que fueron reconstruidos inductivamente a partir de los casos analizados: de oficio, obrera y de emprendimiento estratégico. Se discute la heterogeneidad de las identidades laborales en el retail y el modo como estas están condicionadas por el trayecto biográfico de los trabajadores y por las condiciones de trabajo en las tiendas.
\end{abstract}

Palabras clave: trabajo, identidad laboral, perfil identitario, retail, Chile

\begin{abstract}
The article presents the results of a qualitative study on the labor identities on stores' employees of large and modernized retail companies (supermarkets, department stores, home improvement stores) in Santiago, Chile. The stories of a non probabilistic, purposive and opinatic sample of 18 workers are analyzed, divided into 4 groups according to gender and generation criteria: 5 women and 4 men under 40 years and 5 women and 4 men over 45 years. The information generated through in-depth interviews was analyzed using the holistic-content mode of narrative analysis, looking to rebuild the plot and the overall meaning of the workers' identitary narratives. Three identity profiles are presented that were reconstructed inductively from the cases analyzed: occupational identity, worker identity and strategic-entrepreneurial identity. The heterogeneity of work identities in retail stores is discussed, along with the way they are heavily influenced by the biographic course of workers and by working conditions in the stores.
\end{abstract}

Keywords: work, labor identity, identity profile, retail, Chile

Los procesos de construcción identitaria que llevan a cabo los sujetos en sus espacios laborales constituyen un foco de creciente interés para los estudios psicosociales del trabajo en Chile y América Latina (Battistini, 2004; De la Garza, 2000; Soto, 2008, 2011; Stecher, 2010, 2011). Existe una amplia evidencia acumulada respecto de las transformaciones experimentadas por las empresas y los escenarios de trabajo en el contexto de los procesos de reestructuración productiva y modernización empresarial ocurridos en las últimas tres décadas en la región.

Antonio Stecher, Facultad de Psicología, Universidad Diego Portales, Santiago, Chile.

Los resultados presentados en este estudio son parte de la Tesis Doctoral realizada para la obtención del grado de Doctor en Psicología Social en la Universidad Autónoma de Barcelona. La realización de dicho estudio fue posible gracias al apoyo de la Comisión Nacional de Investigación Científica y Tecnológica del Gobierno de Chile, la Fundación Carolina y la Universidad Diego Portales.

La correspondencia relativa a este artículo debe ser dirigida a Antonio Stecher, Facultad de Psicología, Universidad Diego Portales, Vergara 275, Santiago, Chile. E-mail: antonio.stecher@udp.cl 
Como lo señalan diversos estudios, dichos procesos - que suponen importantes modificaciones en los modos de organización del proceso de trabajo, las relaciones laborales y modalidades de empleo, las formas de organización y gestión empresarial, el patrón tecnológico, las culturas laborales y el perfil de la fuerza de trabajo (De la Garza, 2000; Ramos, 2009) — han impactado fuertemente en los escenarios laborales, transformando los contextos tecno-socio-productivos en los cuales los trabajadores forjan sus experiencias cotidianas y configuran sus narrativas identitarias (Battistini, 2004; Díaz, Godoy \& Stecher, 2005; García \& Carvajal, 2007; PulidoMartínez \& Carvajal, 2001; Sisto, 2009; Soto, 2008, 2011; Stecher, 2010, 2011; Todaro \& Yáñez, 2004).

A pesar del creciente interés en el estudio de esta problemática y de la consolidación de ciertos núcleos de investigación en los últimos años (Díaz et al., 2005; Román \& Avendaño, 2002; Sisto, 2009; Soto, 2008, 2011; Stecher, 2010, 2011), no cabe duda de que, para el caso de Chile, existe un déficit de investigación psicosocial orientada a dar cuenta, específicamente, de los procesos identitarios de particulares colectivos de trabajadores insertos en diferentes sectores productivos y escenarios laborales. Dicha ausencia conlleva muchas veces el riesgo de generalizar la realidad socio-laboral y los procesos identitarios de un particular escenario o condición laboral a la totalidad de los contextos de trabajo y de los trabajadores. También conlleva el riesgo de extrapolar linealmente, sin las mediaciones conceptuales y empíricas requeridas, ciertas reflexiones y resultados de investigaciones producidas en los países nor-atlánticos de industrialización avanzada a la realidad socio-laboral de Chile y América Latina (De la Garza, 2000; Stecher, 2011). La tesis que afirma que en el contexto del nuevo modelo de desarrollo capitalista — global, informacional, post-fordista y en red (Castells, 2001; Ramos, 2009) predominaría un nuevo tipo de identidad laboral basada en los valores del emprendimiento, la flexibilidad, la movilidad y el cálculo estratégico individual, permite ilustrar este punto. Dicha afirmación, que encuentra asidero en estudios empíricos acotados sobre cuadros gerenciales y/o grupos de profesionales jóvenes de modernas empresas en Chile (Díaz et al., 2005; Soto, 2011) y otros países de la región (Szlechter, 2010), así como en diversas reflexiones e investigaciones sobre el mundo del trabajo y las identidades en los países de industrialización avanzada (Bauman, 2000; Boltanski \& Chiapello, 2002; Du Gay, 1996; Elchardus \& Smits, 2008; Sennett, 2000, 2006; Webb, 2004), muchas veces es tomada como una descripción acabada y exhaustiva que daría cuenta de la totalidad de los procesos identitarios de los trabajadores de la región (De la Garza, 2000; Stecher, 2011). Esto contribuye a la errada representación de un mundo del trabajo homogéneo, entre los distintos países y al interior de cada país y sus distintos sectores productivos, en el que se habría consolidado un nuevo y único tipo de identidad laboral de tipo flexible y emprendedor. Dicha representación resulta especialmente problemática para el caso de América Latina, pues desatiende e invisibiliza la histórica heterogeneidad estructural de la matriz productiva y sociocultural de los países de la región, al interior de los cuales han coexistido y coexisten hasta hoy mundos del trabajo sumamente diversos (De la Garza, 2000; Infante \& Sunkel, 2009).

El presente artículo busca contribuir a ampliar el conocimiento sobre los procesos identitarios de colectivos específicos de trabajadores dentro del heterogéneo mundo del trabajo en Chile, visibilizando el modo en que los procesos de construcción de identidad están fuertemente condicionados, no solo por los singulares trayectos biográficos de cada trabajador, sino también por las específicas características tecno-socio-productivas de los escenarios laborales en que se inserta. Específicamente, se presentan los principales resultados de un estudio empírico cualitativo que tuvo como objetivo describir y analizar las identidades laborales de un grupo de trabajadores de baja calificación empleados en las tiendas (supermercados, tiendas por departamento, tiendas para el mejoramiento del hogar) de grandes y modernizadas empresas de la industria del retail en Santiago de Chile. A partir de la presentación de tres perfiles identitarios o tipos de identidad laboral de los operarios y vendedores del retail en Chile, se busca, por un lado, ampliar la comprensión sobre los procesos identitarios de un particular escenario 
de trabajo perteneciente al sector moderno y formal de la economía chilena y, por otro, visibilizar la importancia de estudios empíricos sensibles a la heterogeneidad entre y dentro de los mundos del trabajo, así como el modo en que las particularidades de los escenarios laborales inciden en los procesos de construcción de identidad.

\section{El Concepto de Identidad Laboral: Narrativas y Perfiles Identitarios}

El estudio asumió una perspectiva crítico-interpretativa de investigación (Thompson, 1998) y abordó los procesos identitarios desde los aportes de la psicología cultural (Bruner, 1991; Crosley, 2003), la psicología y sociología narrativa (Smith \& Sparkes, 2008; Somers, 1994) y la sociología francesa del trabajo (Dubar, 1998, 2001). Desde ese marco, se conceptualizó a las identidades laborales como producciones simbólicas articuladas narrativamente que expresan las modalidades específicas en que un trabajador entiende y experimenta su espacio laboral, significa a los otros con los que se relaciona en el trabajo y se define a sí mismo como un actor singular al interior de ese espacio social. Desde esta perspectiva, las identidades laborales pueden ser entendidas como narrativas identitarias a partir de las cuales un trabajador interpreta y da sentido a su experiencia laboral. Es a través de dichas narrativas —entendidas técnicamente como relatos que articulan en una secuencia temporal y en una trama común un conjunto heterogéneo de eventos- que los trabajadores articulan un sentido de sí mismos, una particular modalidad de auto-interpretación de lo que son, de lo que han llegado a ser, de lo que aspiran ser y de lo que son para los otros (Lawler, 2002). La identidad laboral no es, así, una esencia anclada en la persona del trabajador (visión psicologicista) ni un derivado mecánico de la posición que este ocupa en un determinado organigrama o en una determinada categorización socio-ocupacional (visión estructural-funcionalista). Es una construcción simbólica que le permite al trabajador, en un particular escenario laboral, hacer significativas sus acciones, lograr un cierto sentido de distinción, singularidad y continuidad de la experiencia, al mismo tiempo que construir un sentimiento de pertenencia, semejanza e integración social (Sisto, 2009; Thompson, 1998). La construcción de dicha identidad se lleva a cabo en el crisol de las interacciones cotidianas dentro del trabajo y de la movilización de los recursos simbólicos (referenciales identitarios) presentes en el escenario laboral (Battistini, 2004). Pero también puede implicar la movilización de significados diversos (e.g., respecto de sí mismo, del trabajo, de la sociedad) que cada trabajador porta producto de una biografía particular, de una singular historia de experiencias formativas y/o laborales previas, así como de su inserción en otros mundos sociales (familia, amistades, ocio, vida política, por ejemplo) en los que participa. Todos estos aspectos están condicionados por la posición del actor en ciertas categorías socioeconómicas, de género, generación y étnicas dentro de la sociedad (Battistini, 2004). Siguiendo a Dubar (1998, 2001), se asumió que las narrativas identitarias de los trabajadores resultan de la confluencia, muchas veces problemática y tensional, de dos dimensiones: (a) una biográfica y diacrónica, que da cuenta de la imagen de sí mismo como trabajador que el sujeto ha ido construyendo a lo largo de su historia laboral y de vida (identidad para sí) y (b) una relacional y sincrónica, centrada en las modalidades en que el trabajador es reconocido y categorizado en ciertos roles y posiciones por la empresa, las jefaturas e incluso los clientes y compañeros en un escenario laboral específico (identidad para otros).

A partir del análisis de las narrativas identitarias de un conjunto de trabajadores insertos en un particular escenario laboral, es posible reconstruir inductivamente los perfiles identitarios característicos de dicho contexto de trabajo. Dichos tipos de identidad laboral dan cuenta de modalidades prototípicas de narrar e interpretar la experiencia laboral y deben ser entendidos como tipos ideales o aparatos heurísticos que, al mismo tiempo que se originan en los datos empíricos y permiten la generalización de los mismos, no coinciden ni constituyen la representación de ningún caso empírico real (Dubar, 2001). 


\section{La Modernización de la Industria del Retail en Chile y el Trabajo en las Tiendas}

Se denomina industria del retail a los grandes y modernos grupos empresariales del sector del comercio minorista que administran simultáneamente diversas empresas e incluso distintos canales de negocio de venta masiva dentro del rubro - cadenas de supermercados, tiendas por departamento, tiendas para el mejoramiento del hogar y locales especializados (e.g., farmacias, materiales de construcción) - y que, en torno al rubro inicial y principal de venta de bienes y productos, han ido articulando otros nichos de negocio y rentabilidad: retail financiero, negocio inmobiliario, agencias de viaje, desarrollo de marcas propias, entre otros (Calderón, 2006; Fundación Sol, 2011; Gálvez, Henríquez \& Morales, 2009). Se trata de un sector que ha experimentado un profundo proceso de modernización y reestructuración en las últimas dos décadas, llegando a convertirse en uno de los sectores económicos más importantes del país en términos de crecimiento (más de 2.000 locales a lo largo del país), generación de empleo (sobre 260.000 empleos directos), aporte al Producto Interno Bruto, expansión internacional, innovación tecnológica y utilización de diversas estrategias de flexibilidad organizacional externa (en las formas de empleo y en la organización productiva) e interna (temporal, salarial y en la organización del proceso de trabajo) (Calderón, 2006; Cámara de Diputados de Chile, 2007; Centro de Estudios del Retail, 2010; Kalleberg, 2001).

Las tiendas de las grandes empresas del retail se caracterizan por emplear un perfil heterogéneo de trabajadores (jóvenes, de mediana edad y mayores, varones y mujeres, con y sin experiencia laboral previa y con un nivel de estudio desde la educación escolar incompleta a la técnica completa). Dentro de dicha heterogeneidad, predominan las mujeres (60 a 80\% en las tiendas), los jóvenes (60\% menor a 35 años), los trabajadores de baja calificación (aproximadamente $80 \%$ con educación media), sin mayor experiencia laboral previa y pertenecientes a sectores urbano-populares (Cámara de Diputados de Chile, 2007; Fundación Sol, 2011). Se trata de una fuerza de trabajo de baja empleabilidad, ampliamente disponible en el mercado laboral, de bajo costo para las empresas y para la cual las tiendas del retail son unas de las pocas opciones de encontrar un empleo formal. Los empleos de los trabajadores directamente contratados por la empresa, con jornada completa y contratos indefinidos — que fueron el foco del estudio - se caracterizan por ser empleos formales y protegidos, con bajos salarios (entre 1,3 y 2,4 ingresos mínimos mensuales), con importantes asignaciones, bonos y beneficios (escolaridad, salud, descuentos, entre otros), estipulados muchas veces en los instrumentos de negociación colectiva, y con altas tasa de rotación (3,5 años de duración promedio) (Cámara de Diputados de Chile, 2007; Fundación Sol, 2011). En términos de las relaciones laborales, se observa una comparativamente alta tasa de sindicalización (sobre el 50\% en las grandes empresas del sector) y una activa presencia de sindicatos en la mayoría de las tiendas. A pesar de ello, destaca un tipo de relación empresa- trabajadores marcada por una fuerte asimetría, por la desconfianza y por el temor de los trabajadores, por la unilateralidad en la toma de decisiones por parte de las gerencias, por las dificultades para la negociación colectiva y el ejercicio del derecho a huelga y por una alta infraccionalidad laboral de las empresas (Cámara de Diputados de Chile, 2007; Durán \& Kremerman, 2008).

Con respecto a la gestión de la mano de obra y la organización del proceso de trabajo en las tiendas, es posible mencionar la implementación de diversas innovaciones flexibilizadoras por parte de las gerencias: (a) el uso de distintas estrategias de flexibilidad temporal (sistemas de turnos flexibles y variables, trabajo de fin de semana, horas extras, turnos nocturnos) que buscan distribuir la fuerza de trabajo de un modo que se ajuste a las necesidades y demandas variables de los clientes y que tienen un fuerte impacto en la calidad de vida personal y familiar de los trabajadores (Díaz, Ruiz Tagle, Aguilar \& Frías, 1999); (b) la utilización, especialmente en el caso de los vendedores comisionistas de las tiendas por departamento, de diversas modalidades de pago variable orientadas al incremento de la productividad; (c) la polifuncionalidad 
de los empleados y la intensificación de la carga de trabajo (física, cognitiva y emocional); (d) el despliegue de distintos mecanismos de control socio-ideológico orientados a lograr, a través de charlas, revistas corporativas, rituales al inicio de la jornada, capacitaciones e incentivos, que los trabajadores se identifiquen con la empresa, sus valores y el ideal de trabajador flexible, emprendedor, orientado al servicio y fuertemente comprometido con el cliente (Alvesson \& Willmott, 2002); (e) la existencia de mecanismos permanentes de evaluación que conllevan una fuerte carga de responsabilidad individual y una exigencia de auto-activación y autorregulación sobre la fuerza de trabajo; y (f) una fuerte política de capacitación y ciertas posibilidades de movilidad interna y ascenso dentro de la tienda.

Un aspecto distintivo del modelo productivo de las tiendas del retail es que ese conjunto de innovaciones flexibles o post-fordistas coexisten con ciertos principios de racionalización taylorista del trabajo (Stecher, Godoy \& Toro, 2010). Al respecto, es posible destacar la división del espacio de la tienda en secciones o departamentos claramente delimitados y con funciones específicas (por ejemplo, en un supermercado: cajas, verdulería, carnicería, panadería), las que suponen muchas veces diferenciaciones internas en estatus, autonomía y salario de los operarios y vendedores; la estandarización y protocolización del proceso de trabajo en torno a tareas sencillas de ejecución, lo que incluye la uniformización de los intercambios comunicativos entre vendedores y clientes; la organización fuertemente jerárquica y piramidal de la sala de venta; la presencia de fuertes mecanismos de vigilancia y control directo sobre la fuerza de trabajo (jefaturas directas, cámaras, guardias) y el intenso desgaste físico del trabajo (Stecher et al., 2010).

\section{Método}

Se llevó a cabo un estudio cualitativo enmarcado en una perspectiva crítico-interpretativa de la realidad social (Bruner, 1991; Thompson, 1998). Consistentemente con el concepto de identidad laboral, se asumió un enfoque teórico-metodológico narrativo, orientado a la producción y análisis interpretativo de las narrativas identitarias que construyen los actores sociales (Crossley, 2003; Hiles, Cermak \& Chrz, 2009; Lieblich, Tuval-Mashiach \& Zilber 1998).

\section{Participantes}

La muestra, no probabilística de tipo intencional opinático estuvo conformada por 18 trabajadores divididos en cuatro grupos, según género y generación: cinco mujeres y cuatro hombres menores de 40 años y cinco mujeres y cuatro hombres mayores de 45 años. Son empleados asalariados contratados directamente por alguna de las grandes y modernizadas empresas del retail: supermercados (cinco trabajadores, dos empresas y cuatro tiendas), tiendas por departamento (nueve trabajadores, tres empresas y cinco tiendas) y tiendas para el mejoramiento del hogar (cuatro trabajadores, una empresa y tres tiendas). Son trabajadores con más de un año de antigüedad en la tienda, con contrato indefinido y de jornada completa, con formación técnica como máximo nivel educacional concluido, e insertos en los puestos de más bajo nivel jerárquico dentro de los locales (vendedores y operarios sin cargo de jefatura).

Los entrevistados fueron contactados a través de informantes clave y siguiendo una lógica de muestreo secuencial conceptualmente conducido (Flick, 2004), buscando, a medida que se avanzaba en las entrevistas, el análisis y la comparación constante de los casos, abarcar un conjunto diverso de trabajadores de tienda. Se consideró, progresivamente, a trabajadores heterogéneos en aspectos tales como sindicalización, años de trabajo en el retail y la empresa, arreglos familiares (estado civil, hijos), trayectorias laborales previas, empresa y tipo de tienda, local y estatus de la sección o departamento donde se desempeñan. 


\section{Instrumento}

Se utilizó una entrevista individual en profundidad de orientación narrativa, la que buscaba que los entrevistados relataran en forma abierta y en una secuencia temporal su experiencia laboral en las tiendas (ingreso, presente, aspiraciones futuras) (Flick, 2004; Lieblich et al., 1998). Para conducir la conversación se contó con un guión de temas sobre los cuales se solicitaba a los entrevistados profundizar en la medida que aparecían en su relato. El guión incluía aspectos de la vida extra-laboral del sujeto (familia, tiempo libre, participación en colectivos, entre otros) y de su historia de vida (familia de origen, estudios, experiencias laborales previas), que eran abordados en la medida que emergían, a la luz del propio relato laboral del entrevistado, como pertinentes para comprender su experiencia de trabajo en las tiendas.

\section{Procedimiento}

Las entrevistas fueron realizadas en los años 2010 y 2011 por el propio investigador en lugares públicos cercanos al espacio de trabajo o en el hogar del entrevistado. Tuvieron una duración aproximada de dos horas y fueron grabadas en audio y transcritas, previa aprobación y firma por parte de los entrevistados de una carta de consentimiento informado. En esta se señalaban los objetivos del estudio y se garantizaba la confidencialidad y el anonimato en la publicación de la información.

\section{Análisis de Datos}

Se llevó a cabo un análisis narrativo de las entrevistas, tomando como referencia el modelo de análisis narrativo de Hiles et al. (2002) y Lieblich et al. (1998). Cada uno de los relatos fue sometido a un análisis denominado análisis narrativo holístico y centrado en el contenido, cuyo propósito es reconstruir el sentido y la orientación global de cada una de las narrativas de los trabajadores, a través de lecturas sucesivas del texto y de un análisis de los distintos fragmentos o micro historias que lo componen.

Las preguntas centrales que orientaron el análisis fueron: ¿Cuál es la trama general que organiza y articula en una secuencia temporal significativa los elementos heterogéneos y dispersos que aparecen en el relato? ¿Cuáles son las distintas posiciones de identidad en que queda situado el trabajador en tanto protagonista de su relato y cómo en su articulación ellas permiten reconstruir la identidad laboral entendida como narrativa identitaria? (Lawler, 2002).

\section{Resultados}

Se describen e ilustran con testimonios los tres perfiles identitarios predominantes entre los 18 operarios y vendedores de las tiendas del retail, reconstruidos a partir de las narrativas identitarias analizadas.

\section{Perfil Identitario de Oficio: El Trabajo en el Retail Supone Ejercer un Oficio Valorado Pero Fuertemente Amenazado}

Este perfil identitario da cuenta de aquel colectivo de trabajadores que narra y significa su experiencia laboral en las tiendas en términos, fundamentalmente, del desempeño de un oficio en el cual se es experto, en el que se es reconocido por la empresa, con el cual se está fuertemente identificado y cuyo ejercicio cotidiano da sentido al trabajo. Por lo general, este perfil se asocia a trabajadores varones, insertos en las secciones o departamentos de mayor estatus y con mejor remuneración, que tienen sobre 35 años y que llevan largos años desempeñando su oficio. Destacan dos colectivos de trabajadores: los denominados maestros de los supermercados (carnicero, panadero, encargado de pescadería) y los "antiguos" vendedores de tienda por 
departamento. Estos últimos fueron socializados en el imaginario de las antiguas tiendas por departamento de carácter familiar y más exclusivas, donde los vendedores eran bien remunerados, valorados y reconocidos como poseedores de un saber experto.

Este perfil supone una narrativa en la que se marca una distinción entre él como trabajador experto y el colectivo masivo de meros operarios o simples empleados sin experiencia ni calificación; en la que se enfatizan en el relato las actividades cotidianas del oficio que se desempeña, las que aparecen dotadas de valor e importancia para la tienda y los clientes; en la que se afirma el saber experto que se posee y la autonomía que se merece, así como las tensiones que surgen cuando estos son limitados por las jefaturas; en la que se destaca el valor moral del desempeño correcto del oficio, no por adhesión a los intereses de la empresa, sino por un sentido de pertenencia a la comunidad de saberes, deberes y derechos propios del oficio; y en la que, si bien se reconocen ciertos aspectos negativos del tipo de trabajo en las tiendas (sobre todo las extensas jornadas y los turnos de fin de semana), se expresa estar acostumbrado a los mismos:

Yo soy maestro carnicero, llevo 30 años en el supermercado trabajando y de primera mi experiencia fue buena, o sea, yo era chiquillo cuando entré, 17 años, con maestros de 30, y todo fue buena onda. Es que lo mío, yo he sido carnicero toda mi vida. Soy hijo de carnicero (...). Me gusta lo que trabajo en carne. Tuve la posibilidad de trabajar con muy buenos maestros, me enseñaron harto y, a la vez, yo siempre he sido agradecido de ellos. (operario de carnicería, supermercado)

Un aspecto distintivo de este perfil es que se encuentra fuertemente tensionado, producto de los procesos de modernización llevados a cabo por las empresas del retail. Las identidades de oficio - que provienen de la época previa a la reciente modernización y reestructuración de la industria, de las "antiguas y familiares" empresas del retail- han sido fuertemente amenazadas, debido a un conjunto de procesos de reorganización empresarial orientados a la reducción de costos. Entre ellos se pueden destacar los siguientes: (a) la opción por contratar masivamente trabajadores jóvenes sin calificación ni experiencia y por sueldos apenas superiores al salario mínimo; (b) por rescindir o cambiar los contratos de los antiguos trabajadores; (c) por priorizar el autoservicio de los clientes y disminuir la importancia de los vendedores y operarios; (d) por introducir principios de polifuncionalidad que llevan a que un trabajador de oficio tenga que realizar múltiples tareas que exceden su tradicional ámbito de acción; (e) por lógicas neo-tayloristas de estandarización y protocolización de procesos que inhiben la autonomía de los trabajadores y (f) por estrategias de gestión orientadas a diluir las antiguas y bien remuneradas identidades de oficio y reemplazarlas por una única identidad genérica de "colaborador" polifuncional, disciplinado, de bajo costo, sin experiencia e identificado principalmente con la empresa. Como diría Dubar (1991, 1998), se trata de antiguas identidades de oficio que, en el marco de los procesos de reorganización de la industria, han sido bloqueadas y no son ya objeto del mismo reconocimiento, simbólico y material, que tuvieron antaño.

Este contexto permite entender el predominio en este perfil identitario de una estructura temporal y una tonalidad emocional de la narrativa basada en un contrapunto nostálgico y algo desesperanzado entre un pasado que aparece como un paraíso perdido - el antiguo mundo del retail, con empresas familiares pequeñas, con dueños cercanos y presentes, con trabajadores de oficio bien pagados, valorados, respetados, comprometidos, con largos años en la empresa y con cierto estatus dentro y fuera de la tienda-y un presente de profundas transformaciones en las empresas que tensiona o amenaza la identidad laboral cultivada y forjada a lo largo de los años, y que lleva, en el caso de los más jóvenes, a pensar en buscar nuevas oportunidades laborales y, en el caso de los mayores, a una posición de resignación a la espera de la jubilación:

\footnotetext{
...y a el último tiempo, como bajaron el presupuesto, no se puso mayores exigencias, entonces, hemos tenido de todo tipo de vendedores, que no venden nada, que de repente no se han preocupado ni de la presentación (...). La rotación es increíble, ya no hay ese compromiso que uno tiene con la empresa, ese sentido de pertenencia que muchas veces se tenía, de la empresa antigua, la de familia, la que tenía a un trabajador por 20 años, eso ya no se da (...) ahora está lleno de gente nueva, gente con un contrato nuevo, mucho joven (...) y pagándoles muy poca plata. (vendedor, tienda por departamento)
} 


\section{Perfil Identitario Obrero: El Trabajo en el Retail es un Trabajo Exigente y Sacrificado que Permite Subsistir y Mantener a la Familia}

En este perfil el trabajo en el retail es significado como "un mero trabajo". Más allá de las especificidades de la labor desempeñada en las tiendas o de las particularidades del sector comercio, lo fundamental es que se trata de un empleo que permite obtener un ingreso, subsistir y mantener al grupo familiar. La definición de sí que se articula simbólicamente es la de un hombre o mujer trabajadora, padre o madre de familia que debe luchar y esforzarse cada día por llevar el sustento al hogar. Dicho en otros términos, los trabajadores asociados a este perfil se caracterizan por hablar de su empleo en el retail como un caso particular de una experiencia más amplia y general: la necesidad de trabajar en la vida para poder subsistir y salir adelante.

En general, este perfil se asocia a puestos menos calificados, de menor estatus y remuneración dentro de las tiendas, a trabajadores que tienen responsabilidades familiares y que no cuentan con credenciales educativas como para obtener un mejor empleo, de carácter formal, en el mercado laboral. Se establece una clara distinción entre los trabajadores, por un lado, y las jefaturas y la empresa, por otro, no existiendo una identificación con la cultura corporativa. Existe una clara conciencia de ser parte del eslabón más bajo de las tiendas, de pertenecer a un colectivo desprotegido y explotado, que es considerado por la empresa como mera fuerza de trabajo anónima y fácilmente reemplazable. Se destaca el modo en que el trabajo en las tiendas supone una exigencia permanente, un fuerte control y vigilancia, bajos salarios, alta demanda de jefaturas y clientes y disponibilidad total para responder a los turnos variables y extensos que, además de generar agotamiento físico, socavan la vida familiar. Es decir, se destacan todos aquellos aspectos ligados a una representación del trabajo como desgaste, sacrificio y explotación, lo que explica la preeminencia en esta narrativa de un sentimiento de frustración, malestar, cansancio y falta de reconocimiento:

\footnotetext{
Me costó acostumbrarme a estar de pie, es muy sacrificado, porque son 10 horas que nosotros estamos ahí. (...) De repente uno ya está tan cansado, así en la última [como] que actúa como por inercia, ya los pies no dan más. (...) Es cansador el hecho de la presión de estar [en la tienda], es como una máquina, (...) estamos embrutecidos. (...) Llego a la casa, me siento, estoy un rato con mi nieta ahí, trato de jugar con ella un ratito, pero estoy re-cansado, me duelen los pies. Me dan ganas de pintar, a mí me gusta pintar o dibujar, pero no lo puedo hacer, porque estoy muy cansado, o sea, me duelen mucho los pies, la espalda, entonces, no lo puedo hacer, no puedo desarrollar esa parte de mi vida. (vendedor, tienda para el mejoramiento del hogar)
}

Estos sentimientos aparecen más acentuados en las trabajadoras mujeres, por la doble carga de trabajo — productiva y reproductiva - que deben asumir. Si el trabajo cotidiano desgastante y exigente en las tiendas tiene un valor y un sentido para este perfil, es, básicamente, porque permite cumplir con la función e identidad de padre o madre proveedor, la que aparece revestida de un fuerte valor moral. La estructura temporal de la narrativa en este perfil se organiza a partir de la referencia a un pasado en que una determinada situación vital de crisis de empleo, falta de recursos o la llegada de un hijo obliga a salir a buscar (un nuevo) trabajo, constatando que, dada la exigua calificación y experiencia, el retail aparece como una de las pocas posibilidades de empleo formal que garantice un ingreso regular mensual y ciertas protecciones básicas para la familia (e.g., salud, previsión social). El retail es algo a lo que se ha llegado por necesidad, no un empleo en que se realice una vocación, como en el caso del perfil de oficio. El presente laboral está marcado por la experiencia de desgaste, aguante, sacrificio y lucha. El futuro aparece teñido de un sentimiento de resignación e impotencia, pues, sobre todo en los trabajadores mayores, se visualizan pocas posibilidades de encontrar un mejor empleo en otro sector o lograr un ascenso en la tienda.

Lo anterior no implica una actitud de displicencia respecto de las tareas asignadas en la tienda. Estas se cumplen en general con esmero y dedicación, debido a la importancia que tiene en este perfil la ética protestante/industrial del trabajo y su mandato de compromiso y respon- 
sabilidad del trabajador, con independencia, hasta cierto punto, de las condiciones del trabajo que se ejerce. Esta particular interpretación de la experiencia laboral tampoco significa que no se valoren determinados aspectos del trabajo en las tiendas, más allá del sentido básicamente instrumental del mismo. Se destacan como aspectos positivos el carácter formal del empleo en una gran empresa (contrato, beneficios y protección social), los aprendizajes en el día a día y en las capacitaciones (las que son especialmente valoradas, dado el bajo nivel de calificación asociado a este perfil), la posibilidad que brinda de hacer algo útil y productivo ante sí mismo y la sociedad y, muy especialmente, las relaciones cotidianas e informales de amistad y compañerismo con los colegas, las que son significadas como un refugio que permite amortiguar las duras exigencias del trabajo y disfrutar de momentos de camaradería, complicidad y encuentro con otros.

\title{
Perfil Identitario de Emprendimiento Individual Estratégico: El Trabajo en el Retail Es una Competencia y Carrera por la Obtención de Nuevas Oportunidades y Beneficios
}

En este perfil el empleo en la tienda es significado como una etapa transitoria, como un momento puntual dentro de una carrera individual, en la que el foco y la meta están puestos en ir progresivamente obteniendo nuevos beneficios y oportunidades, ascendiendo dentro de la empresa o el mercado laboral y mejorando permanentemente el estatus, el ingreso y la calidad de las condiciones de trabajo. El valor y sentido del puesto que se ocupa en la tienda están fuertemente ligados al modo en que este es funcional a ese proyecto estratégico de movilidad y mejora continua. Este perfil aparece asociado fundamentalmente a trabajadores jóvenes, con un nivel de formación más alta que el promedio — estudios técnicos_-, habitualmente insertos en secciones o departamentos de mayor estatus y que suelen haber tenido ya algún ascenso dentro de la tienda (o haber recibido la promesa de que lo recibirán pronto) que conlleva alguna mejora de las condiciones de trabajo. En sus narrativas identitarias los trabajadores vinculados a este perfil tienden a hablar mucho más de sus competencias, habilidades, redes y potencialidades que de su puesto de trabajo y sus labores cotidianas en la tienda. El foco del relato no es el ejercicio de un oficio o las condiciones y duras exigencias del trabajo cotidiano, sino el propio trabajador como agente individual inserto en un contexto en que debe saber moverse y situarse para destacar sobre los otros, aprovechar las oportunidades y los vínculos y ser visto y valorado por las jefaturas, de tal modo de ir avanzando en pos de sus objetivos de movilidad:

\begin{abstract}
Me gustaría estar en otra área trabajando de lunes a viernes, no todos los días. (...) Si se puede [seguir en la misma empresa] sí, pero si no, cualquier otra empresa que esté trabajando, que gane plata y que esté bien conmigo mismo. (...) Me gustaría seguir surgiendo. (...) Mi idea este otro año es irme a la central a otro cargo, pero no quiero seguir más en ventas, porque el horario me aburrió (...). Igual sé que si no sigo trabajando acá, no soy tonto y puedo conseguir pega en otro lado (...). Tengo buenos contactos en la misma empresa, en las mismas marcas, tengo tarjeta de todos los supervisores de todas las marcas. (...) Para promotor o supervisor de las marcas, como llevo tanto tiempo aquí, puedo trabajar en otra parte, tengo contactos, gracias a Dios. (vendedor, tienda por departamento)
\end{abstract}

En este perfil hay un énfasis en el buen desempeño y en el cumplimiento de los objetivos asignados por las jefaturas. Ello, sin embargo, no está ligado a una identificación con los valores de un oficio, la ética del trabajo o los intereses de la empresa, sino más bien a la adhesión al proyecto personal de movilidad que exige destacar en la tienda y sobresalir sobre el resto. De este modo, no existe en este perfil una relación de identificación fuerte con la empresa o su cultura corporativa, sino, más bien, una relación instrumental en la que el trabajador sabe que, así como es utilizado y exigido al máximo por la compañía, también tiene derecho a utilizar y aprovechar a la empresa como plataforma de su proyecto personal de movilidad y ascenso laboral.

En términos de la estructura temporal de la narrativa, este perfil se caracteriza por aludir a un pasado laboral que es descrito enfatizando sus aspectos negativos e inferiores al actual 
puesto que se detenta en la tienda; por resaltar el modo en que se logró obtener el actual puesto dentro de la tienda, a partir de una suma de situaciones en que la decisión y voluntad del trabajador jugaron un rol importante; y por destacar la manera en que se está actuando estratégicamente para obtener lo antes posible un nuevo y mejor puesto dentro de la organización. Es esa dinámica de movilidad la que organiza el relato de este perfil, en el cual el sujeto queda posicionado como un agente individual activo, calculador, estratégico y orientado hacia el futuro. Esto permite entender el predominio de un sentimiento de bienestar con lo logrado y de expectación ansiosa respecto de la posibilidad de alcanzar el nuevo objetivo que se tiene en mente, así como la presencia de un cierto desazón o aburrimiento respecto al día a día laboral, en tanto es difícil identificarse y comprometerse con una labor que es vista como un paso provisorio hacia algo mejor.

\section{Discusión}

El estudio presentado, a pesar de sus limitaciones vinculadas al reducido tamaño de la muestra y al riesgo de reificación de los procesos identitarios que supone la construcción de tipologías, permite destacar ciertos aspectos de los procesos de construcción de identidad de los trabajadores del retail en Chile.

Un primer aspecto a relevar es la heterogeneidad de perfiles identitarios presentes en los trabajadores de las tiendas. Más allá de condiciones de trabajo y empleo que, a primera vista y en términos generales, podrían ser consideradas como relativamente similares, los trabajadores del retail constituyen un colectivo heterogéneo en términos del modo en que narran y significan su empleo en las tiendas, se definen a sí mismos, a la empresa y a sus compañeros, dotan de sentido a su trabajo cotidiano y se proyectan laboralmente hacia el futuro. Este hallazgo permite cuestionar aquellas tesis que, basadas en estudios con profesionales jóvenes de grandes empresas en Chile y/o en la extrapolación de estudios de los países de industrialización avanzada, afirman que, bajo las condiciones del capitalismo flexible, existiría en Chile y América Latina una única o principal forma de subjetividad laboral y que esta sería el fiel reflejo del ideal de trabajador emprendedor, competitivo, flexible, polivalente y autoregulado, propio del imaginario laboral post-fordista (Bauman, 2000; Sennett, 2000). Como lo sugieren los hallazgos de este estudio, las identidades laborales, incluso al interior del sector moderno y formal de la economía, parecen ser más heterogéneas de lo que muchos de esos discursos anuncian. Si bien los valores y referentes identitarios del imaginario post-fordista juegan un rol importante en el espacio laboral de las tiendas del retail, como lo expresa el perfil emprendedor-individualista, en ningún caso constituyen las únicas matrices simbólicas en dicho contexto de trabajo, siendo inadecuado reducir la problemática de las identidades laborales en dicha industria, o en otras industrias o sectores productivos en Chile, al análisis del modo en que dicho ideal de trabajador flexible se habría ido expandiendo y encarnando en las últimas décadas.

Un segundo aspecto a destacar es el modo en que dicha diversidad de tipos de identidad laboral surge de la confluencia de los dos ejes de análisis mencionados: por un lado, la particular representación de sí mismo que ha construido un trabajador a lo largo de su biografía personal y laboral (identidad para sí) y, por otro, el modo en que es reconocido y categorizado por la empresa, los clientes y los colegas en el proceso de trabajo en las tiendas (identidad para otro) (Dubar, 1998). Dado que a las tiendas del retail llegan trabajadores diversos (en términos de edad, género, formación, responsabilidades familiares, experiencia laboral previa) y que la gerencia, a su vez, los ubica y categoriza en puestos de relativamente diferente estatus, autonomía, responsabilidad y remuneración, es posible entender que emerjan —en el cruce de esos dos ejes- narrativas y perfiles identitarios diferentes. Es solo atendiendo a esa dialéctica simbólica de auto-reconocimiento (cómo se reconoce a sí mismo en tanto trabajador a la luz de su historia personal y laboral) y hetero-reconocimiento (cómo es reconocido y categorizado por la empresa a partir del particular lugar que ocupa dentro del proceso de trabajo de la tienda) que es posible comprender las narrativas identitarias de los actores laborales (Jenkins, 2000). 
Lo anterior exige una reconstrucción detallada, tanto de los trayectos biográficos y las experiencias laborales pasadas y presentes del trabajador y sus anhelos futuros, como del particular contexto organizacional y productivo en que se encuentra inserto. Así, por ejemplo, el perfil obrero del retail solo puede ser comprendido atendiendo, por un lado, a la presencia de un trabajador que se define a sí mismo, a la luz de su particular biografía, como un luchador, como padre o madre proveedor, como sujeto sin mayores calificaciones y para quien el empleo en el retail, como cualquier otro empleo, es parte de una batalla incesante y desgastante por la subsistencia; así como, por otro lado, atendiendo a su particular inserción en la tienda donde ocupa los puestos de menor estatus, autonomía, reconocimiento y remuneración, donde es sometido a turnos flexibles que se le imponen y afectan su vida familiar y donde experimenta fuertes y permanentes presiones, exigencias y controles que le devuelven una imagen de sí mismo como mero engranaje desechable y anónimo de una máquina productiva que lo desgasta y explota.

Por último, los resultados y argumentos presentados dan cuenta de la necesidad de desarrollar nuevos estudios empíricos que amplíen nuestra comprensión sobre los procesos de construcción de identidades laborales en Chile y América Latina, analizando para ello las narrativas identitarias que elaboran los trabajadores, así como atendiendo a los procesos socio-simbólicos de auto y hetero-reconocimiento que están a la base de las mismas. Estas dos dimensiones permiten una lectura sensible, tanto de las singularidades de las biografías de los individuos y de sus trayectorias laborales como de las particulares características tecno-socio-productivas de los escenarios laborales en los que están insertos, muchos de los cuales han experimentado importantes cambios en las últimas décadas en el marco de los procesos de reestructuración productiva y modernización empresarial. Como lo ilustra el estudio presentado, esta perspectiva puede contribuir a visibilizar la heterogeneidad de los procesos identitarios en los contemporáneos y transformados mundos del trabajo.

\section{Referencias}

Alvesson, M. \& Willmott, H. (2002). Identity regulation as organizational control: Producing the appropriate individual. Journal of Management Studies, 39, 619-644. doi:10.1111/1467-6486.00305

Battistini, O. (Comp.). (2004). El trabajo frente al espejo: continuidades y rupturas en los procesos de construcción identitaria de los trabajadores. Buenos Aires, Argentina: Prometeo.

Bauman, Z. (2000). Trabajo, consumismo y nuevos pobres. Barcelona, España: Gedisa.

Boltanski, L. \& Chiapello, E. (2002). El nuevo espíritu del capitalismo. Madrid, España: Akal.

Bruner, J. (1991). Actos de significado. Madrid, España: Alianza.

Calderón, A. (2006). El modelo de expansión de las grandes cadenas minoristas chilenas. Revista de la CEPAL, 90, $151-170$.

Cámara de Diputados de Chile (2007). Informe de la Comisión Investigadora de la Dirección del Trabajo en la fiscalización del cumplimiento de la legislación laboral en el rubro de los supermercados. Valparaíso, Chile: Autor.

Castells, M. (2001). La era de la información. Vols. I y II. Madrid, España: Alianza.

Centro de Estudios del Retail (2010). Calidad de servicio en la industria del retail en Chile: caso supermercados. Santiago, Chile: Autor/Universidad de Chile.

Crossley, M. L. (2003). Formulating narrative psychology: The limitations of contemporary social constructionism. Narrative Inquiry, 13, 287-300. doi:10.1075/ni.13.2.03cro

De la Garza, E. (Coord.) (2000). Tratado latinoamericano de sociología del trabajo. México, DF, México: El Colegio de México/Facultad Latinoamericana de Ciencias Sociales/Universidad Autónoma Metropolitana.

Díaz, X., Godoy, L. \& Stecher, A. (2005). Significados del trabajo, identidad y ciudadanía: la experiencia de hombres $y$ mujeres en un mercado laboral flexible (Cuadernos de Investigación $\mathrm{N}^{\mathrm{o}} 3$ ). Santiago, Chile: Centro de Estudios de la Mujer.

Díaz, E., Ruiz Tagle, J., Aguilar, O. \& Frías, P. (1999). Jornada de trabajo en el sector comercio: la experiencia en grandes tiendas, supermercados y pequeñas tiendas en malls (Cuadernos de Trabajo $\mathrm{N}^{\circ}$ 12). Santiago, Chile: Dirección del Trabajo, Departamento de Estudios.

Dubar, C. (1991). La socialisation: Construction des identités sociales et professionnelles [La socialización: construcción de identidades sociales y profesionales]. Paris, Francia: Armand Colin.

Dubar, C. (1998). Trajetórias sociais e formas identitárias: alguns esclarecimentos conceituais e metodológicos [Trayectorias sociales y formas identitarias: algunos esclarecimientos conceptuales y metodológicos]. Educação \& Sociedade, 19(62), 13-30. doi:10.1590/S0101-73301998000100002

Dubar, C. (2001). El trabajo y las identidades profesionales y personales. Revista Latinoamericana de Estudios del Trabajo, 13, 5-16.

Du Gay, P. (1996). Consumption and identity at work. London, Reino Unido: Sage. 
Durán, G. \& Kremerman, M. (2008). Caracterización del sector retail: una mirada general (Cuaderno de Investigación $\mathrm{N}^{\mathrm{o}}$ 7). Santiago, Chile: Fundación Sol.

Elchardus, M. \& Smits, W. (2008). The vanishing flexible: Ambition, self-realization and flexibility in the career perspectives of young Belgian adults. Work, Employment and Society, 22, 243-262. doi:10.1177/09500170080891 03

Flick, U. (2004). Introducción a la investigación cualitativa (1ºd.). Madrid, España: Morata.

Fundación Sol (2011). La modernización de la industria del retail en Chile. Santiago, Chile: Autor.

Gálvez, T., Henríquez, H. \& Morales, G. (2009). Holding y tiendas: la trama que multiplica las ventas y redobla el trabajo (Cuaderno de Investigación $\mathrm{N}^{\circ} 36$ ). Santiago, Chile: Dirección del Trabajo.

García, C. M \& Carvajal, L. M. (2007). Tecnologías empresariales del yo: la construcción de sujetos laborales en el contexto del trabajo inmaterial. Universitas Psychologica, 6, 49-58.

Hiles, D., Cermak, I. \& Chrz, V. (2009). Narrative oriented inquiry: A dynamic framework for good practice. En D. Robinson, P. Fisher, T. Yeadon-Lee, S. J. Robinson \& P. Woodcock (Eds.), Narrative, memory, and identities (pp. 53-65). Huddersfield, Reino Unido: University of Huddersfield.

Infante, R. \& Sunkel, O. (2009). Chile: hacia un desarrollo inclusivo. Revista de la CEPAL, 97, 135-154.

Jenkins, R. (2000). Categorization: Identity, social process and epistemology. Current Sociology, 48(3), 7-25. doi:10.11 $77 / 0011392100048003003$

Kalleberg, A. L. (2001). Organizing flexibility: The flexible firm in a new century. British Journal of Industrial Relations, 39, 479-504. doi:10.1111/1467-8543.00211

Lawler, S. (2002). Narrative in social research. En T. May (Ed.), Qualitative research in action (pp. 242-258). London, Reino Unido: Sage.

Lieblich, A., Tuval-Mashiach, R. \& Zilber, T. (1998). Narrative research: Reading, analysis, and interpretation. London, Reino Unido: Sage.

Pulido-Martínez, H. C. \& Carvajal, L. M. (2001). Discurso y construcción de identidad: el caso de los propietarios de vehículos del transporte urbano en Bogotá. Debates en Psicología, 4, 91-103.

Ramos, C. (2009). La transformación de la empresa chilena: una modernización desbalanceada. Santiago, Chile: Universidad Alberto Hurtado.

Román, J. A. \& Avendaño, C. (2002). Metáforas para re-pensar al sujeto en la sociedad del empleo: voces desde Barcelona. Psykhe, 11(2), 181-196.

Sennett, R. (2000). La corrosión del carácter: las consecuencias personales del trabajo en el nuevo capitalismo. Barcelona, España: Anagrama.

Sennett. R. (2006). La cultura del nuevo capitalismo. Barcelona, España: Anagrama.

Sisto, V. (2009). Cambios en el trabajo, identidad e inclusión social en Chile: desafíos para la investigación. Universum, 24(2), 192-216.

Smith, B. \& Sparkes, A. C. (2008). Contrasting perspectives on narrating selves and identities: An invitation to dialogue. Qualitative Research, 8, 5-35. doi:10.1177/1468794107085221

Somers, M. R. (1994). The narrative constitution of identity: A relational and network approach. Theory and Society, 23, 605-649. doi:10.1007/BF00992905

Soto, A. (Ed.) (2008). Flexibilidad laboral y subjetividades: hacia una comprensión psicosocial del empleo contemporáneo. Santiago, Chile: LOM/Universidad Alberto Hurtado.

Soto, A. (2011). Narrativas de profesionales chilenos sobre sus trayectorias laborales: la construcción de identidades en el trabajo. Psykhe, 20(1), 15-27. doi:10.4067/S0718-22282011000100002

Stecher, A. (2010). El análisis crítico del discurso como herramienta de investigación psicosocial del mundo del trabajo: discusiones desde América Latina. Universitas Psychologica, 9, 93-107.

Stecher, A. (2011). Transformaciones del trabajo y procesos identitarios en el "nuevo" capitalismo: notas para una discusión en el contexto latinoamericano. En B. Medrado \& W. C. Galindo (Org.), Psicologia social e seus movimentos: 30 anos de ABRAPSO [Psicología social y sus movimientos: 30 años de ABRAPSO] (pp. 207-232). Recife, Brasil: Universidade Federal de Pernambuco.

Stecher, A., Godoy, L. \& Toro, J. P. (2010). Condiciones y experiencias de trabajo en la sala de venta de un supermercado: explorando los procesos de flexibilización laboral en el sector del retail en Chile. Polis, 27, 523-550.

Szlechter, D. (2010). ¿Gerentes o empresarios? Apuntes para un debate teórico sobre el trabajo en empresas mutinacionales. Psicoperspectivas, 9(2), 180-201.

Thompson, J. B. (1998). Ideología y cultura moderna. México, DF, México: Universidad Autónoma Metropolitana.

Todaro, R. \& Yáñez, S. (Eds.) (2004). El trabajo se transforma: relaciones de producción y relaciones de género. Santiago, Chile: Centro de Estudios de la Mujer.

Webb, J. (2004). Organizations, self-identities and the new economy. Sociology, 38, 719-738. doi:10.1177/0038038504 045861

Fecha de recepción: Abril de 2012.

Fecha de aceptación: Agosto de 2012. 